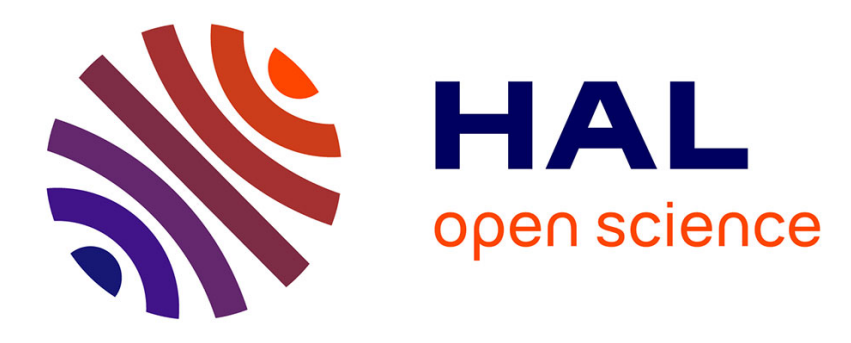

\title{
Hellequin bi-frons : à propos du sacré, du comique et du théâtral
}

\author{
Karin Ueltschi
}

\section{To cite this version:}

Karin Ueltschi. Hellequin bi-frons : à propos du sacré, du comique et du théâtral. Connochie-Bourgne, Chantal (dir.). Façonner son personnage au Moyen Âge, 53, Presses universitaires de Provence, pp.329338, 2007, Senefiance, 9782821836136. 10.4000/books.pup.2303 . hal-02990299

\section{HAL Id: hal-02990299 https://hal.science/hal-02990299}

Submitted on 5 Nov 2020

HAL is a multi-disciplinary open access archive for the deposit and dissemination of scientific research documents, whether they are published or not. The documents may come from teaching and research institutions in France or abroad, or from public or private research centers.
L'archive ouverte pluridisciplinaire HAL, est destinée au dépôt et à la diffusion de documents scientifiques de niveau recherche, publiés ou non, émanant des établissements d'enseignement et de recherche français ou étrangers, des laboratoires publics ou privés. 


\section{Hellequin bifrons : À propos du sacré, du comique et du théâtral}

Paru dans Façonner son personnage au Moyen Age, Études réunies par Chantal Connnochie-Bourgne, Senefiance $n^{\circ} 53$, Publications de l’Université de Provence, 2007, p. 329-338.

Hellequin n'est jamais le personnage principal d'une oeuvre, peut-être parce qu'il est fondamentalement ambivalent : tantôt, il semble personnifier le diable et la mort à la tête d'un cortège de revenants, tantôt c'est un personnage loufoque et comique dans un défilé carnavalesque. S'il est l'un ou l'autre, jamais les deux à la fois, une parenté souterraine existe entre ces deux visages opposés, établissant une corrélation entre la problématique de la mort et celle de l'amour et des noces. La présente investigation voudrait examiner comment le personnage se constitue et se diversifie notamment par le biais de sa théâtralisation, donne naissance, à travers des fusions thématiques et mythiques, à de nouveaux types littéraires célèbres, le tout dans une articulation originale du sacré et du comique.

\section{Un personnage incertain}

Hellequin est une figure aux contours mal esquissés et variable comme peut l'être la forme d'un nuage évoluant dans le vent : ce n'est d'ailleurs pas par hasard que certains critiques pensent qu'il tire son origine d'une réaction "primitive » face aux brumes vaporeuses montant du sol, des traînés de brouillard, ou encore de feu-follets et de manifestations orageuses ${ }^{1}$. Il faut bien chercher pour rassembler une trentaine d'occurrences qui le nomment dans la littérature médiévale ; il vit surtout de manière allusive, nameless, dans un certain anonymat, avant de surgir en tant que personnage à part entière une fois que la littérature aura apprivoisé son essence mythique sauvage, et au prix de certaines métamorphoses, à commencer par le nom.

Lorsque nous étudions les attestations les plus anciennes du nom de Hellequin, cette impression d'incertitude se concrétise. Voici ses principaux noms, la plupart du temps au génitif ou au cas régime absolu puisque il se se manifeste en général entouré de sa mesnie: Familia Herlechini (Orderic Vital, 1135), milites Herewini (Pierre de Blois, 1175), Herla et familia Herlethingi (Gautier Map, 1182/93), militia / familia Hellequini (Hélinand de Froidemont, 1229/30), Hellequin (Guillaume d'Auvergne, 1231/36), familia Herlequini (Codex Runensis, XIII'), familia Allequini Etienne de Bourbon, av. 1261) pour les occurrences latines. Et en français : Mesnie Hellequin (Philoména, XII ${ }^{2}$ ), mesniée Hellequin (Huon de Méry, 1240), Herlekin (Miracle de Saint Eloi, XIII'), masnée Herllequin (Roman de Confession, XIII ${ }^{2}$ ), Hel(l)equin (Luque la Maudite, $\mathrm{XIII}^{2}$ ), maisinie Hielekin / roy Hellekin (Jeu de la Feuillée, 1276), maisnie Hellekin (Roman de Fauvel, 1310/1314), mesgnée Hanequin / Helequin (Robert le Diable, $\mathrm{XIV}^{2}$ ), mesnie Helquin (Doctrine chrétienne, $\mathrm{XIV}^{2}$ ), mesnie Hellekin (Mariage des filles au diable), mesgnie Hennequin (Chronique de Normandie, $\mathrm{XV}^{\mathrm{e}}$ ).

On constate donc de grandes variations graphiques et phonétiques au niveau du nom du personnage, variations dont aucune n'a pu s'imposer au détriment des autres, ce qui confirme l'étoffe sinon ténue du moins fuyante de la figure. Parle-t-on vraiment d'un seul et même personnage ? Les constantes circonstancielles semblent l'étayer ; une stature générique, stylisée du personnage semble s'imposer dès ce point de départ, au sens où l'entend J. Ribard,

\footnotetext{
${ }^{1}$ Cf. O. Driesen, Der Ursprung des Harlekin. Ein kulturgeschichtliches Problem, Berlin, Alexander Duncker, 1904, p. 66-67.
} 
qui y associe à une fonction mythique ou sociale ${ }^{2}$. C'est d'ailleurs grâce à ces constantes circonstancielles qu'il est possible de découvrir Hellequin dans bien des passages.

A cette pluralité de noms s'ajoute l'hésitation concernant sa morphologie : est-ce un géant ou un nain ? Les géants et les nains, représentant tous deux une manifestation anormale de l'humanité, possèdent en effet une parenté paradoxale confirmée par d'anciennes traditions. En outre, au Moyen Age un « jaiant» ne désigne pas forcément une créature de taille immense : c'est simplement «une race primordiale» dont la morphologie est « incertaine $»^{3}$. Aussi bien les nains que les géants ont partie liée avec le monde surnaturel, et tout particulièrement avec le monde souterrain, ce qui apporte enfin un élément positif à la carte d'identité de notre figure. Depuis l'Antiquité, la gigantomachie est en particulier associée aux manifestations volcaniques ; Euripide localise le pays des géants sur l'Etna ${ }^{4}$. Or, le volcan constitue la bouche de l'enfer ${ }^{5}$; certains géants « ne craignent pas d'affirmer leur parenté avec Satan ${ }^{6} »$. Cette vocation mythique et surnaturelle se confirme ainsi dans la coïncidence des figures du nain et du géant - et de Hellequin - avec certains traits de l'homme sauvage, le passeur par excellence - au même titre que le Janus antique, le gardien bifrons des portes - du temps et du passage.

Cette identité fluctuante se complique à travers la multiplicité des fonctions que notre personnage peut assumer : si la plupart du temps il est le meneur d'un cortège, donc un guide et un commandeur, il est tantôt présenté comme un chevalier guerrier, tantôt comme un chasseur, voire comme un roi, et son cortège assimilé respectivement à une troupe militaire ou à un groupe de chasseurs. Dans l'exemple suivant, les deux fonction coïncident :

Et l'on rapporte que de semblables faits sont arrivés dans les forêts de Grande Bretagne ou de Petite Bretagne : les gardes des forêts (que le peuple appelle forestiers, c'est-à-dire les gardiens des filets des chasseurs, des réserves de gibier ou des bois royaux) racontent qu'ils voient très souvent, certains jours, vers l'heure de midi et dans la première partie de la nuit, quand brille la pleine lune, une compagnie de chevaliers qui chassent, dans le vacarme des chiens et des cors. A ceux qui les questionnent, ils répondent qu'ils sont de la suite et de la maison d'Arthur ${ }^{7}$.

Si le thème de l'affrontement militaire est toujours une question de vie ou de mort, celui de la chasse a également vocation à orienter le récit soit vers le tragique, soit vers le surnaturel à travers sa finalité profonde, la mort ${ }^{8}$. Or, cette fonction de mise à mort - rituelle il est vrai s'applique tout spécialement à une figure qui émerge peu à peu dans ces contextes, le veneur infernal, Satan, constamment à la recherche de nouvelles proies pour peupler son enfer: «Venator diabolus, in cuius figura Nemrod ille gigas venator coram Domino, ut in Genesi ; venatores pravi homines, ut in propheta: Venantes ceperunt me, quasi avem, inimici mei

\footnotetext{
${ }^{2}$ « Tous ces personnages (...) portent en fait un nom, mais c'est un nom générique. Ils s'identifient à leur fonction, qu'elle soit sociale ou mythique. Ils sont de ce fait exemplaires et le sens qu'il faut leur attribuer, dans une lecture de type à la fois structural et symbolique, ne soulève pas en général de difficulté (...). J. Ribard, Le moyen âge, littérature et symbolisme, Paris, Champion, 1984, p. 75.

${ }^{3}$ C. Lecouteux, Démons et génies du terroir au Moyen Age, Imago, 1995, p. 35

${ }^{4}$ Ch. Cusset, «L'Etna dans Le Cyclope d'Euripide, in D. Bertrand (dir.), Mythologies de l'Etna, Presses Universitaires Blaise Pascal, coll. «Volcaniques », Clermont-Ferrand, 2004 p. 25.

${ }^{5}$ Voir notre article «Sybille, Arthur et sainte Agathe : les monts italiens comme carrefour des autres mondes », à paraître in Materiali arturiani nelle letterature di Provenza, Spagna, Italia, Alessandria, Ed. dell' Orso, 2005.

${ }^{6} \mathrm{~F}$. Dubost, Aspects fantastiques de la littérature narrative médiévale, XIIe-XIIIe siècles, Paris, Champion, 1991, p. 588

${ }^{7}$ Gervais de Tilbury, Gervais de Tilbury, Otia imperalia,(III, n $\left.{ }^{\circ} 58\right)$, éd. G.W. Leibniz, in Scriptores rerum Brunsvicensium, I, Hanovre, 1707, p. 987 et sq. Trad. française par A. Duchesne, Le Livre des Merveilles, Les Belles Lettres, 1992, p. 152.

${ }^{8}$ F. Dubost, op. cit., p. 335 et p. 349
} 
gratis $^{9} »$, dit Raban Maur. Pour Saint Augustin, le diable est le «pessimus venator mundi ${ }^{10}{ }$ ! Hellequin, grâce aux particularités circonstancielles de ses apparitions, se coule tout naturellement dans ce moule identitaire dans certains textes cléricaux, ce qui se répercutera naturellement sur sa destinée future.

Si cette assimilation au diable semble ainsi être le trait identitaire qui émerge et se détache, dès les plus anciens textes vernaculaires, cette identification semble contestée par la présence d'attributs fonctionnels diamétralement opposés: Hellequin peut apparaitre comme une figure burlesque, carnavalesque. Certaines de ses parures comme les clochettes peuvent rappeler la frivolité comme chez Huon de Méry dans Le Tournoi de l'Antéchrist (v.686$689)^{11}$ où le tintement des clochettes de Coïntise évoque à l'auteur Hellequin ! Dans Le Jeu de la Feuillée $e^{12}$, Hellequin amoureux de la fée Morgane est une créature inoffensive et à vrai dire absente puisqu'il est seulement évoqué par son serviteur Croquesot qui le précède, ce qui est peut-être une manière de dire la difficulté d'ériger en personnage une figure mythique. Le Roman de Fauvel ${ }^{13}$ fait subir à Hellequin une dépréciation burlesque comparable. Cependant, certains textes vernaculaires comme Luque la Maudite ${ }^{14}$ qui relate le mariage (ou rapt en enfer) du diable Hellequin avec Luque, conservent sans la résoudre cette ambivalence du personnage. La question reste posée : qu'y a-t-il caché sous la cape de Hellequin ? Quelle est la valeur de ses attributs dans la définition des traits de son caractère?

\section{Une tête ou un masque ?}

Lorsque dans le Conte du Papegau, on veut enlever le heaume d'un chevalier noir que le héros - Arthur - a tué, on s'aperçoit avec stupeur que le heaume et la tête sont une seule et même chose. Le masque est le visage.

Le masque le plus rudimentaire, c'est la barbe. Dans les différents avatars de Hellequin, la famille sémantique autour de hure, hérupé, hurepiaus est récurrente, mots désignant soit la tête, soit les poils de bêtes qui mordent ${ }^{15}$. Tout se passe comme si chevelure et barbe devaient se rejoindre pour dissimuler le visage sous une masse poilue indifférenciée. Or, la bouche de l'enfer, dans le théâtre médiéval, est représentée par une gueule diabolique appelée la «hure », et le rideau qui la ferme s'appelle « la chape de Hellequin »! La barbe possède des référents symboliques vastes, constituant notamment des métonymies courantes de leur porteur. On connaît cette espèce particulièrement nocive des géants coupeurs de barbes tels qu'ils apparaissent notamment dans le Tristan de Thomas ou dans Meriadeuc!

La cape et la coule peuvent se voir attribuer une fonction «masquante » semblable, même si à première vue il s'agit simplement du costume des moines. Mais elles peuvent servir d'abri à des créatures plus suspectes. Orderic Vital, peignant le cortège de Hellequin, parle de clercs ou de moines portant une cape ou un capuchon (cappis, cucullis). Hélinand de Froidemont, quelques années plus tard, évoque un revenant qui lui aussi porte une cape dont le poids équivaut à celui de ses péchés. La mention de la cape et/ou du couvre-chef semble

\footnotetext{
${ }^{9}$ Raban Maur, De rerum nat., VIII, I, CXI, 226.

${ }^{10}$ Sermon LI, PL XXXVIII, 334. Cité in J. Voisenet, Bêtes et Hommes dans le monde médiéval. Le bestiaire des clercs du Ve au XIIe siècle, Turnhout, Brepols, 2000, p. 221.

${ }^{11}$ Huon de Méry, Le Tournoi de l'Antéchrist, éd. G.Wimmer, S. Orgeur, Paradigme, 1994.

12 Adam de la Halle, Euvres Complètes, éd. P.Y. Badel, Le Livre de Poche, coll. Lettres Gothiques, 1995.

${ }^{13}$ Le Roman de Fauvel de Gervais du Bus, éd. Lanfors, Paris, 1914-19 (SAFT 63).

${ }^{14}$ K. Meisen, Die Sagen vom wütenden Heer und wilden Jäger, Verlag Aschendorf, Münster in Westfalen, 1935 (Volkskundliche Quellen, Heft 1), p. 62-69.

${ }^{15}$ Ph. Walter, Canicule. Essai de mythologie sur Yvain de Chrétien de Troyes, Paris, SEDES, 1988, p. 85-116.
} 
donc avoir une signification ancienne reliant directement le motif de la mascarade à celui de la mort, du diable et à Hellequin ${ }^{16}$ !

Le chapeau ${ }^{17}$ serait lié depuis un temps immémorial à des divinités ayant trait à la vie après la mort (Fortleben). Ainsi trouve-t-on volontiers toutes sortes de fantômes affublés de grands chapeaux. Wodan en particulier (dont certains critiques ont fait un référent de Hellequin) apparaît volontiers avec un chapeau à large bord, raison pour laquelle on l'appelle Sîdhöttr, le Breithütige. Autre variante intéressante, le meneur de la chasse sauvage peut ne pas avoir de tête du tout, ou alors posséder un chef de la taille d'un poing ${ }^{18}$ : ainsi, la capuche ou la coule peuvent également dissimuler une absence, tout aussi monstrueuse que la représentation la plus effrayante. Et comment saisir la stature précise du personnage s'il ne possède pas de chef ! Ajoutons que pour certains critiques, notre motif peut être rapproché des Männerbünde germaniques dont les représentants se caractérisent par leurs visages noircis et leurs capuches bleues ou noires, figurant des puissances chtoniennes, voire des personnifications d'esprits de mort ${ }^{19}$, et dont l'aspect théâtral est à souligner.

Or, dans de nombreux contextes où apparaît Hellequin ou un de ses avatars, donc avant même sa constitution en véritable personnage, de curieuses questions sont alors posées par des personnages divers à propos de la capuche. Etienne de Bourbon introduit le thème en évoquant une famille démoniaque qui va à cheval et qui se complimente fièrement en disant : «ce capuchon me sied bien $^{20}$ ! » Cette exclamation se transforme ensuite en interrogation et devient récurrente dans le sillage de Hellequin. Chez Adam de la Halle, dans son Jeu de la Feuillée, Croquesot demande Me siet il bien li hurepiaus (v. 590) ?; Jean Dufournet qui souligne l'élément mystérieux aux échos poétiques inquiétants parle de "formule magique » encadrant la féerie ${ }^{21}$. Citant un célèbre passage des Merveilles de Rigomer où le héros doit affronter toute une armée de créatures en cape et coule, Philippe Ménard évoque de son côté un «pouvoir magique ${ }^{22}$ » du capuchon baissé, qui aussitôt relevé fait perdre leurs moyens aux invincibles combattants. Otto Driesen, en comparant les occurrences de la question rituelle chez Etienne de Bourbon et chez Adam de la Halle constate : "En ce qui concerne le fond, l'une et l'autre question se rapportent à la tête du Herlekin. Donc, la tête est une caractéristique des démons appartenant au type de Herlekin ${ }^{23}{ }^{2}$. Ce critique va même jusqu'à traduire le me siet il bien li hurepiaus par Ma tête me va-t-elle bien ${ }^{24}$ ? Gustave Cohen va dans ce sens lorsqu'il propose que la question pourrait « se rapporter à une tête de mort, à un

\footnotetext{
${ }^{16}$ Cf. J.C. Schmitt, Le corps, les rites, les rêves, le temps. Essais d'anthropologie médiévale, Paris, Gallimard, 2001, p. 216.

${ }^{17}$ Et plus particulièrement les espèces suivantes: Breithut, Langhut, Schlapphut, et Blauhütel. A. Endter, Die Sage vom wilden Jäger und der wilden Jagd. Studien über den deutschen Dämonenglauben, InauguralDissertation, Seinhausen, 1933, p. 22.

${ }^{18}$ Ibid., p. 33.

${ }^{19}$ G. Widengren, « Harlekintracht und Mönchskutte, Clownhut und Derwischmütze“, in Orientalia Suecana, II, fas. 2/4, Upsala, 1963, p. 89.

${ }^{20}$ Etienne de Bourbon, Tractatus de diversis materiis predicabilius, éd. F. Lecoy, 1877. In Anecdotes historiques, légendes et apologues tirés du recueil inédit d'Etienne de Bourbon, Paris, 1877, p. 322.

${ }^{21} \mathrm{~J}$. Dufournet, Adam de la Halle à la recherche de lui-même ou le jeu dramatique de la Feuillée, SEDES, 1974, p.134.

${ }^{22} \mathrm{Ph}$. Ménard, «Une parole rituelle dans la chevauchée fantastique de la 'Mesnie Hellequin' », in Littératures. Mélanges offerts au Professeur René Fromilhague, Publications de 1'Université de Toulouse - Le Mirail, $n^{\circ}$ 910, printemps 1984, p. 7.

${ }^{23}$ O. Driesen, op. cit., p. 65 . Toutes les traductions sont de notre fait.

24 "Die merkwürdige Frage der Herlekins, ob ihnen der Kopf gut stehe." O. Driesen, op. cit., p. 66.
} 
masque complet »; il se pourrait que dans « cette chevauchée des morts », le hurepiaus « ait recouvert une tête de diable ${ }^{25} »$.

Dans des passages où subsistent seulement des traces de la question rituelle, le lien avec la mascarade est quasiment affirmée : Et adoncques Richard sans Paour demanda a Hellequin comment ilz povoyent avoir telle figure trouvee laquelle il portoient car il sembloit proprement qu'ilz fussent hommes tous vifz, et Hellequin luy respondit que quant ilz devoyent errer ilz trouvoient mainte chose par le vouloir de Dieu ${ }^{26}$. Autrement dit, la question posée stipule que l'apparence que les gens se donnent n'est pas leur vrai visage, mais un visage d'emprunt qu'ils " portent» comme l'on porte un masque, en l'occurrence un masque d'hommes vivants, alors qu'ils sont des revenants, contradiction sur laquelle porte précisément la question de Richard. D'après ce texte, la question rituelle concernerait donc la nature de la personne interrogée, sa qualité de vivant ou de mort. Nous pourrions multiplier les exemples. Dans la perspective qui est la nôtre, c'est bien une question-clef.

\section{De la forêt sur les planches}

C'est sans doute grâce à l'acclimatation de notre figure d'abord à l'iconographie, puis au théâtre qu'elle va sortir peu à peu des brumes mythiques et se constituer en personnage. Cet aspect théâtral, il est vrai, peut être souligné dès l'occurrence la plus ancienne, chez Orderic Vital, qui peint le cortège funèbre de la famille Hellequin défilant devant un spectateur affolé et contraint : il a été déniché de derrières les néfliers où il avait cru pouvoir se cacher. Un texte comme le mariage de Fauvel va exploiter cet aspect théâtral du cortège, à travers les 78 miniatures que comporte le manuscrit ${ }^{27}$. Hellequin y apparaît tantôt comme un cheval, tantôt comme un centaure ou un géant. Ces représentations rendent compte du caractère fondamentalement théâtral inhérent aux apparitions de Hellequin, d'abord personnification et incarnation du masque, donc par définition biface ${ }^{28}$, incarnation de l'altérité, du signe renvoyant à quelque chose d'autre que lui-même.

On sait qu'il existe une parenté entre sacré, théâtre et comique. Déjà en Grèce antique, «le masque de théâtre n'est-il pas lié à un rite d'évocation de la mort ${ }^{29}$ »? En partant des derviches, de l'église syrienne et des ascètes de l'église orientale qui portaient une longue cape maintes fois reprisée, on peut remonter au costume porté par les mimes de l'Antiquité, le centunculus, confectionné lui aussi à partir de plusieurs morceaux de tissu. L'acteur qui portait ce costume avait un rôle comique, celui du stupidus ${ }^{30}$. Mais il était le stupidus parce qu'à travers son costume et son caractère il renvoyait au rusticus, au paysan. Cette longue robe était l'habit des pauvres ${ }^{31}$. C'est précisément la raison pour laquelle les ascètes chrétiens auraient très tôt adopté ce costume en signe de pauvreté et d'humilité. Il en va de même en ce qui concerne la capuche pointue : à l'origine, elle appartenait à la caste des paysans aussi bien en Italie qu'en Grèce, puis semble également avoir connu la déviation vers une utilisation

\footnotetext{
${ }^{25}$ G. Cohen, « Un terme de scénologie médiévale et moderne : chape d'Hellequin - manteau d'Arlequin », in Mélanges de philologie romane et de littérature médiévale offerts à Ernest Hoepffner, Paris, Les Belles Lettres, 1949 , p. 115.

${ }^{26}$ Richart sans Paour de Gilles Corrozet, éd. D. J. Conlon (Richard sans Peur), Chapel Hill, North Carolina Studies in the Romance Languages and Literatures, U.N.C. Department of Romance Languages, 1977. chap. 5 , p. 76.

${ }^{27}$ M. Lecco, Il motivo della Mesnie Hellequin nella letteratura medievale, Turin, Edizioni dell'Orso, 2001, p. 89 et sq.

${ }^{28}$ J.-C. Schmitt, op. cit, 2001, p. 218.

${ }^{29} \mathrm{Ph}$. Walter, Le Bel Inconnu de Renaut de Beaujeu. Rite, mythe et roman, Paris, PUF, 1996, p. 99.

${ }^{30}$ G. Widengren, art. cit, p. 44-45.

${ }^{31}$ Cf. Apulée, Les Métamorphoses, éd. D.S. Robertson et trad. P. Vallette, Paris, « Les Belles Lettres », 1985 VII, 5. Cf. aussi I, 6 et IX, 12 et 30.
} 
comique, pourvue sans doute très tôt de clochettes. Puis, les moines occidentaux ont à leur tour adopté cette coiffe pour s'identifier aux petites gens comme par exemple saint François d'Assise dont les disciples continuent de porter le titre de ioculatores dei ou ioculatores domini $^{32}$ ! Dans le premiers temps, on les a ainsi considérés comme fous, stulti. Et Geo Widengren d'ajouter non sans malice qu'il suffit de considérer le franciscain François Rabelais pour que tout soit dit au sujet de l'art de prédication de cet ordre par rapport à la problématique du sacré et du théâtral! Une tradition s'est par la suite institutionnalisée à partir de Barletta (+ 1470) qui aurait introduit le fameux nescit predicare qui nescit barlettare, intégrant dans la prédication un élément théâtral résolument léger. Ainsi, « la ténacité par laquelle l'élément théâtral comique maintient sa position dans la sphère sacrée a quelque chose à nous enseigner au sujet des frontières entre le sacré et le grotesque ainsi que le comique ${ }^{33} »$.

Un autre élément peut être évoqué à ce propos, «l'enfer comique » : «Assurément, le Moyen Age a connu l'épouvante du Diable, la hideur fascinante des tentations et l'atmosphère de la 'chasse aux sorcières'; mais il a souffert aussi de cette obsession insupportable dans les cas extrêmes. Un besoin vital de compensation a joué, obscurément sans doute, mais réellement ${ }^{34}$. » Le côté drolatique du Diable a été exploité notamment dans les «diableries » des mystères, où les diables cabriolent et font les clowns sur la scène. Le sacré, loin de s'opposer au théâtre, peut en faire partie intégrante. Le rire comme les grincements de dents peuvent être subordonnés au même registre.

Hellequin, puisqu'il est masqué, puisqu'il parade, va évoluer dans des directions différentes tout en gardant son noyau identitaire fondamental le reliant aux enfers, plus exactement le positionnant à la frontière des deux mondes. Dans les cortèges macabres qui commencent à orner les murs des cimetières et des églises à partir du XIVe siècle, la Mort entraîne en ricanant le monde entier dans sa danse à la fois rituelle et grotesque. Nous avons à nouveau affaire à une mascarade à travers la mise en scène soigneusement élaborée des attributs de la mort. Or, la Danse macabre n'est rien d'autre qu'une « forme christianisée et moralisée de la mesnie Hellequin ${ }^{35}{ }$. Le dialogue entre les vivants et les morts, le « danseur » qui vient entraîner le vivant dans sa farandole et qui bien entendu ne le lâchera plus, la ronde infernale, le défilé : toutes ces composantes essentielles de la Mesnie Hellequin s'y retrouvent. La mise en rapport avec la peste accentue encore les liens entre les deux motifs : "Lorsqu'au Moyen Age éclate une épidémie, généralement désignée par le nom de peste, elle fait souvent suite à des apparitions de revenants ${ }^{36}$. » En effet, les morts de la Danse, quel que soit le réalisme de leur morphologie, ne sont pas de véritables morts : ils ne sont pas étendus, immobiles, en proie à la décomposition. Au contraire, ils s'agitent ; ils possèdent à la fois les aptitudes humaines habituelles et surnaturelles, diaboliques en particulier puisqu'ils ne viennent pas chercher leurs victimes pour les conduire au paradis : «Le trait de génie fut de mêler les morts aux vivants. Qui donc osa le premier réaliser ce cauchemar ${ }^{37}$ ?»

Puis, notre figure mythique fait un grand bond pour continuer sa danse et son vol à travers ses culbutes sur des planches, avec sa hure stylisée en demi-masque noir, ses haillons en costume de losanges et sa massue transformée en batte, en devenant pour le coup un personnage à part entière au nom désormais immortel, mais dont certaines consonances rappellent toujours l'ancêtre Hellequin: Arlequin, dans sa première apparition, en 1558

\footnotetext{
${ }^{32}$ G. Widengren, art. cit, p. 80.

${ }^{33}$ G. Widengren, art. cit, p. 79.

${ }^{34}$ J. Frappier, Histoire, Mythes et symboles, Genève, Droz, 1976, p. 133.

${ }^{35} \mathrm{Ph}$. Walter, « La Mesnie Hellequin. Mythe calendaire et mémoire rituelle », in Iris, $\mathrm{n}^{\circ} 18$, Centre de recherche sur l'imaginaire, Université de Grenoble 3, 1999, p. 65.

${ }^{36}$ C. Lecouteux, Au-delà du merveilleux. Des croyances au Moyen Age, Paris, Presses de l'université de ParisSorbonne, 1995, p. 113.

${ }^{37}$ E. Mâle, L'Art religieux de la fin du Moyen Age en France, Paris (1908), Armand Colin, 1995, P. 362.
} 
intitulé Histoire plaisante des faits et gestes de Harlequin ${ }^{38}$, ne cesse pas pour autant de s'assimiler implicitement au diable :

Harlequin je m'appele, en qui or tu peux voir

Que les diables n'ont pas plus que moy de scavoir.

Devenu Arlequin, il garde toujours « sur scène quelque chose qui rappelle le passage furieux dans le vent, traduit par les sauts fantastiques qui le distinguent traditionnellement ${ }^{39}$ ». Son habit en losanges rappelant le centunculus des anciens paysans, puis comédiens, puis moines stigmatiserait aussi son caractère diabolique si on se réfère aux travaux de Michel Pastoureau sur le rayét ${ }^{40}$; sa batte possède par ailleurs une fonction éminemment carnavalesque, équivalant «à la marotte du fou de carnaval ou à la vessie que le Pétassou porte à sa ceinture $^{41}$.» Arlequin est un exemple accompli de personnage littéraire et théâtral d'une popularité universelle, aux antipodes du nocturne Hellequin évanescent que nous avons cherché à cerner au début de cette investigation. Arlequin fera surtout rire : ses liens avec le monde des morts se font de plus en plus discrets. Mais parfois, on peut le voir avec une hotte sur le dos, hotte remplie d'enfants, comme Hellequin dans Fauvel, lointain souvenir du ravisseur d'âmes qu'il a initialement étét2.

Interrogeons-nous pour finir sur l'éventuelle stature allégorique de Hellequin dont on a parfois fait une personnification de la mort ; cette démonstration tentait de l'étayer, Hellequin est aux antipodes de l'allégorie. L'étoffe de Hellequin est mythique. L'allégorie implique une schématisation réductrice du personnage: on pourrait dire qu'on donne un signifiant abstrait et conceptuel à une entité concrète. Dans le mythe, le rapport est exactement inversé : nous trouvons une multitude de signifiants autour d'un signifié certes donné, mais polymorphe, mais évanescent. Ainsi, notre figure initialement « anti-personnage » va connaître par la suite une fécondité littéraire paradoxale et absolument inouïe : au niveau des scenarii, au bout de fusions mythémiques et littéraires naturellement, nous retrouvons Hellequin dans la configuration d'un purgatoire ambulant, de la danse macabre et de nos défilés de Carnaval. Quant au personnage, il survit non seulement à travers Arlequin, mais aussi Don Juan, le Juif Errant, Faust et jusqu'au Roi des Aulnes.

Voici donc notre gardien des portes devenu une créature à têtes multiples tout en restant fondamentalement passeur; le théâtre lui-même en effet «revêt des sens mystiques, parce qu'il représente le lieu de passage entre deux mondes ${ }^{43} »$, qui ne sont pas seulement les sphères respectives des spectateurs des acteurs. C'est un lieu précisément qui possède à la fois un paradis et un enfer ! Et c'est en Enfer le plus profond que Dante et Virgile rencontrent le géant Lucifer, ogre infernal : "Quel ne fut pas mon grand étonnement lorsque je vis trois faces à sa tête ${ }^{44}$ !» Une trame littéraire véhiculant un motif ancien peut «noyer», «engloutir» celui-ci, grâce à une «prolifération d'effets secondaires »; dans cette

\footnotetext{
${ }^{38}$ O. Driesen, op. cit., p. 13. Texte reproduit en annexe p. 248 et sq.

${ }^{39}$ G. Widengren, art. .cit., p. 85.

${ }^{40}$ M. Pastoureau, L'étoffe du diable. Une histoire des rayures et des tissus rayés, Paris, le Seuil, 1991.

${ }^{41}$ M. Orsino, «Aspects et métamorphoses du mythe de l'homme sauvage dans le 'Théâtre italien' : Arlequin », in Théâtre et Drame musical, Société Internationale d'Histoire Comparée du Théâtre, de l'Opéra et du Ballet, $\mathrm{n}^{\circ}$ 1, 2003, p. 11-29, p. 19-20.

${ }^{42}$ A. Nicoll représente deux Arlequins porteurs de hotte, p. 142-143 : «Harlequin and his brood », woodcut, tiré de Compositions de rhétorique, Lyon, BN, Paris; « Harlequin brings the children home to their real father », sixteenth-century woodcut, tiré du Recueil de Fossard, XVII, National Museum, Stockholm.

${ }^{43}$ T. Revol, Représentations du sacré dans les textes dramatiques des XIe-XIIIe siècles en France, Paris, Champion, 1999, p. 393.

44 Dante Alighieri, La Divine Comédie, présentée et traduite par D.M. Garin, Paris, Editions de la Différence, 2003, l'Enfer, XXIV, v. 28-38.
} 
perspective, le rôle de la satire ou de l'humour et essentiel ${ }^{45}$. Or, le comique n'est jamais une donnée «brute », mais constitue toujours une appréciation par rapport à quelque chose: « le rire est une volonté de nier, de suspendre et de dominer son effroi ; son émotion, sa piété, sa pitié » : ainsi, le rire peut être « une réaction de défense de l'homme ${ }^{46}$ », en particulier devant la figure la plus effrayante qui puisse se présenter à lui : celle du Grand Passeur.

Karin Ueltschi

${ }^{45}$ D. Poirion, Le merveilleux dans la littérature française du Moyen Age, PUF, 1982, p. 50.

${ }^{46}$ H. Rey-Flaud, Pour une dramaturgie du Moyen Age, PUF, 1980, p. 93. 